\title{
Desafios no processo de educação inclusiva para crianças com transtorno do espectro autista
}

\author{
Challenges in the inclusive education process for children with autism spectrum disorder
}

Desafíos en el proceso de educación inclusiva para niños con trastorno del espectro autista

Lucilla Vieira Carneiro ${ }^{1 *}$, Vitória Polliany de Oliveira Silva1, Fernanda Lima de Vasconcellos Farias ${ }^{1}$, Kátia Suely Queiroz Silva Ribeiro1.

\section{RESUMO}

Objetivo: Analisar os desafios no processo da educação inclusiva para crianças com transtorno do espectro autista. Métodos: Trata-se de um estudo descritivo, do tipo revisão integrativa da literatura, realizado nas bases de dados LILACS, MEDLINE (via BVS) e SCIELO, a partir das palavras-chave: "autismo", "inclusão" e "escola". Resultados: Foram resgatados 54 artigos, e apenas 7 constituíram a amostra final. Os artigos utilizados são de estudos desenvolvidos no Brasil, Austrália e Zimbábue, e evidenciaram que os desafios no processo de educação inclusiva para crianças com TEA, estão relacionados principalmente a características estruturais das escolas, carência de tecnologia de ensino adequada e falta de qualificação dos professores para atenderem esse público. Considerações finais: $O$ processo de inclusão escolar das crianças com TEA impõe a necessidade de adequações no ambiente escolar, no que diz respeito à estrutura física da escola $e$ quantidade de alunos por turma, bem como no que tange à capacitação dos professores para lidarem com essas crianças e adotarem estratégias pedagógicas facilitadoras da aprendizagem das mesmas.

Palavras-chave: Autismo, Inclusão, Escola.

\section{ABSTRACT}

Objective: Analyzing the challenges in the process of inclusive education for children with autism spectrum disorder. Methods: This is a descriptive study, of the type of integrative literature review, conducted in the databases LILACS, MEDLINE (via VHL) e SCIELO, based on the keywords: "autism", "inclusion" and "school". Results: Fifty-four articles were recovered, and only 7 constituted the final sample. The articles used are from studies developed in Brazil, Australia and Zimbabwe, and showed that the challenges in the process of inclusive education for children with ASD are mainly related to structural characteristics of schools, lack of adequate teaching technology and lack of qualification of teachers to serve this public. Final considerations: The process of school inclusion of children with ASD imposes the need for adaptations in the school environment, about the physical structure of the school and the number of students per class, as well as about the training of teachers to deal with these children and adopt pedagogical strategies that facilitate their learning.

Keywords: Autism, Inclusion, School.

\section{RESUMEN}

Objetivo: Analizar los desafíos en el proceso de educación inclusiva para niños con trastorno del espectro autista. Métodos: Se trata de un estudio descriptivo, del tipo de revisión de la literatura integrativa, realizado en las bases de datos LILACS, MEDLINE (vía VHL) y SCIELO, basado en las palabras clave: "autismo", "inclusión" y "escuela". Resultados: Se recuperaron cincuenta y cuatro artículos, y sólo 7 constituyeron la

1 Universidade Federal da Paraíba (UFPB), João Pessoa - PB. *E-mail: lucilla.vc@hotmail.com 
muestra final. Los artículos utilizados provienen de estudios desarrollados en Brasil, Australia y Zimbabwe, y mostraron que los desafíos en el proceso de educación inclusiva para los niños con TEA están relacionados principalmente con las características estructurales de las escuelas, la falta de tecnología de enseñanza adecuada y la falta de capacitación de los maestros para servir a este público. Consideraciones finales: El proceso de inclusión escolar de los niños con TEA impone la necesidad de adaptaciones en el entorno escolar, con respecto a la estructura física de la escuela y el número de estudiantes por clase, así como con respecto a la formación de los profesores para tratar con estos niños y adoptar estrategias pedagógicas que faciliten su aprendizaje.

Palabras clave: Autismo, Inclusión, Escuela.

\section{INTRODUÇÃO}

O transtorno do espectro autista (TEA) é um grupo de distúrbios do desenvolvimento neurológico de início precoce, caracterizado por comprometimento das habilidades sociais e de comunicação, além de comportamentos estereotipados (SILVA M e MULICK JA, 2009). Embora definido por estes principais sintomas, o fenótipo dos pacientes com TEA pode variar muito, abrangendo desde indivíduos com deficiência intelectual (DI) grave e baixo desempenho em habilidades comportamentais adaptativas, até indivíduos com quociente de inteligência (QI) normal, que levam uma vida independente. Estes indivíduos também podem apresentar uma série de outras morbidades, como hiperatividade, distúrbios de sono e gastrintestinais, e epilepsia (CUNHA E, 2015).

Apesar da escassez dos dados epidemiológicos nos países em desenvolvimento, o parâmetro mais utilizado atualmente é do Center for Disease Control, que revelou no Brasil uma prevalência de uma para 68 crianças com TEA, ou seja, uma taxa de 1,47\% (CHRISTENSEN DL, et al., 2016). De acordo com o Ministério da Educação, no Brasil, o número de alunos com TEA matriculados em classes comuns ampliou 37,27 \% em um ano (BRASIL, 2019).

A alta prevalência de diagnósticos de TEA pode estar relacionada com a maior sensibilidade dos instrumentos utilizados para diagnóstico, embora esses recursos possam ser insuficientes para diagnosticar as especificidades do TEA e assim produzir falsos positivos. Deve-se também levar em conta o aumento de serviços de referência que trabalham com a população que possui TEA, como o Centro de Atenção Psicossocial Infantil (CAPSi) e o Centro Especializado em Reabilitação (CER), que contribuem para o aumento de diagnóstico dessa população. E ainda, a ampliação do conhecimento sobre o TEA entre profissionais da saúde que trabalham com essa população, educadores e a comunidade em geral (HILL AP, et al., 2014; BRASIL, 2012).

O movimento para inclusão de crianças com Necessidades Educativas Especiais (NEE) na escola tem ocorrido mundialmente. Desde a década de 1990, com a Declaração de Jomtien, também conhecida como Declaração Mundial de Educação para Todos, juntamente com a Convenção de Direito da Criança e a Declaração de Salamanca estabeleceu-se que toda pessoa (criança, jovem e adulto) deveria usufruir das oportunidades educacionais voltadas às suas necessidades de aprendizagem. Assim, como as pessoas com deficiência requerem atenção especial, devem ser tomadas medidas que garantam a igualdade de acesso à educação a elas como parte integrante do sistema educacional (UNESCO, 1994).

No Brasil, o governo criou políticas e diretrizes que proporcionaram as condições de acesso aos espaços e aos recursos pedagógicos necessários à inclusão. Além disso, viabilizavam ferramentas que apoiam os profissionais na atuação e na compreensão da inclusão escolar, bem como no processo de organização da aprendizagem com vistas à valorização das diferenças, de forma a atender às necessidades educacionais dos alunos. Tais políticas incentivam a formação de professores para o atendimento especializado das crianças com deficiência, além de programas de incentivo da participação da família e das comunidades na escola (BRASIL, 2021).

De acordo com Lima SM e Laplane ALF (2016), a inclusão escolar é uma das políticas que tem promovido, nas últimas décadas, a escolarização de todos os alunos. Os documentos internacionais e a legislação 
brasileira têm contribuído para difundir o conceito e normatizar as práticas inclusivas, que envolvem, de um modo geral, o ensino regular, a Educação Especial e as instâncias públicas e privadas.

Nessa perspectiva, a educação inclusiva é uma modalidade do sistema educacional que promove ensino e aprendizagem na rede de ensino regular para pessoas com deficiência, transtorno global de desenvolvimento e altas habilidades, visando o desenvolvimento de suas potencialidades de acordo com características e necessidades individuais (CAMARGO EP, 2017).

As instituições com Educação Infantil propõem-se a executar ações que promovam o desenvolvimento integral da criança, de modo a contemplar os aspectos cognitivos, físicos e socioemocionais. Desse modo, são necessários elementos que forneçam meios para sua realização, tais como estrutura arquitetônica adequada, capacitação profissional, suporte pedagógico, conscientização e sensibilização dos profissionais, além da participação da família e toda comunidade escolar. Embora respaldada por leis e políticas públicas, prevendo igualdade no acesso e na permanência na escola, a inclusão de pessoas com deficiência no ensino regular evidencia dificuldades que precisam ser superadas, para que o processo possa ser realizado de forma integral e satisfatória no Brasil (CASTRO GG, et al., 2018).

Corroborando com os autores supracitados, Thiengo ER, et al. (2021), afirmam que a saída para a inclusão requer preparo, políticas públicas incisivas e adaptações ambientais para o recebimento desses alunos. De acordo com Adurens FDL e Vieira CM (2018), no censo escolar do Instituto Nacional de Estudos e Pesquisas Educacionais Anísio Teixeira (INEP), referente ao ano de 2016, é possível verificar que $57,8 \%$ das escolas no Brasil têm alunos que frequentam aulas regulares para grupos-alvo de educação especial, em comparação com $31 \%$ em 2008. No entanto, ainda não há garantia de sua persistência na educação formal e da oportunidade de receber educação de qualidade.

Neste contexto, o desenvolvimento deste trabalho justifica-se pela necessidade de compreender como a educação inclusiva pode contribuir no processo educativo de uma criança com TEA, na perspectiva de atender às necessidades individuais de forma inclusiva. $O$ objetivo deste estudo foi analisar os desafios no processo da educação inclusiva para crianças com transtorno do espectro autista.

\section{MÉTODOS}

Trata-se de um estudo descritivo, do tipo revisão integrativa da literatura. Assim, emergiu como questão norteadora: quais as dificuldades no processo de inclusão em relação ao acesso e permanência da criança com transtorno do espectro autista na escola regular?

Para a construção deste estudo foram consultadas as seguintes bases de dados: Literatura LatinoAmericana e do Caribe em Ciências da Saúde (LILACS), Medical Literature Analysis and Retrieval System Online (MEDLINE via BVS) e Scientific Eletronic Library Online (SCIELO). Para realizar a busca, foram utilizadas combinações com as seguintes palavras-chave: "autismo", "inclusão" e "escola", unidas por meio do operador booleano "AND".

Foram incluídos artigos completos relacionados ao objeto de pesquisa, sem restrição de idiomas, indexados e publicados nos referidos bancos de dados nos últimos dez anos. Os critérios de exclusão para a amostra foram: artigos do tipo revisão de literatura e duplicados nas bases de dados.

O processo de elaboração dessa revisão foi constituído por seis etapas: Identificação do tema e elaboração da questão norteadora da pesquisa, Estabelecimento de critérios para inclusão e exclusão de estudos e busca na base de dados, Categorização dos estudos selecionados, Avaliação dos estudos incluídos na revisão, Análise e interpretação dos resultados, Apresentação da revisão e síntese do conhecimento (MENDES KDS, et al., 2008).

Para sistematizar o processo seleção dos artigos optou-se pela metodologia Preferred Reporting Items for Systematic Reviews and Meta-Analyses (PRISMA), que apresenta critérios que devem ser utilizados em revisões bibliográficas ao longo do estudo (LIBERATI A, et al., 2009). As etapas deste processo estão descritas na forma de um fluxograma, evidenciando desde o resgate dos artigos nas bases de dados até sua posterior inclusão (Figura 1). 
Figura 1 - Fluxograma do processo de seleção dos artigos baseado no método PRISMA.

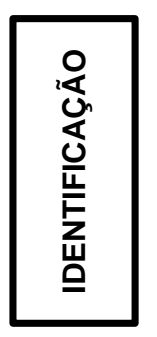

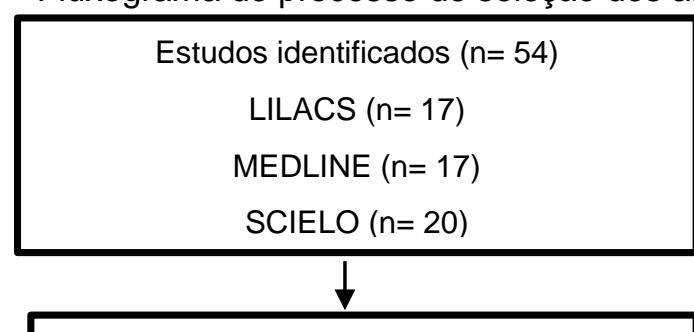

Total de artigos após remoção dos duplicados $(n=52)$
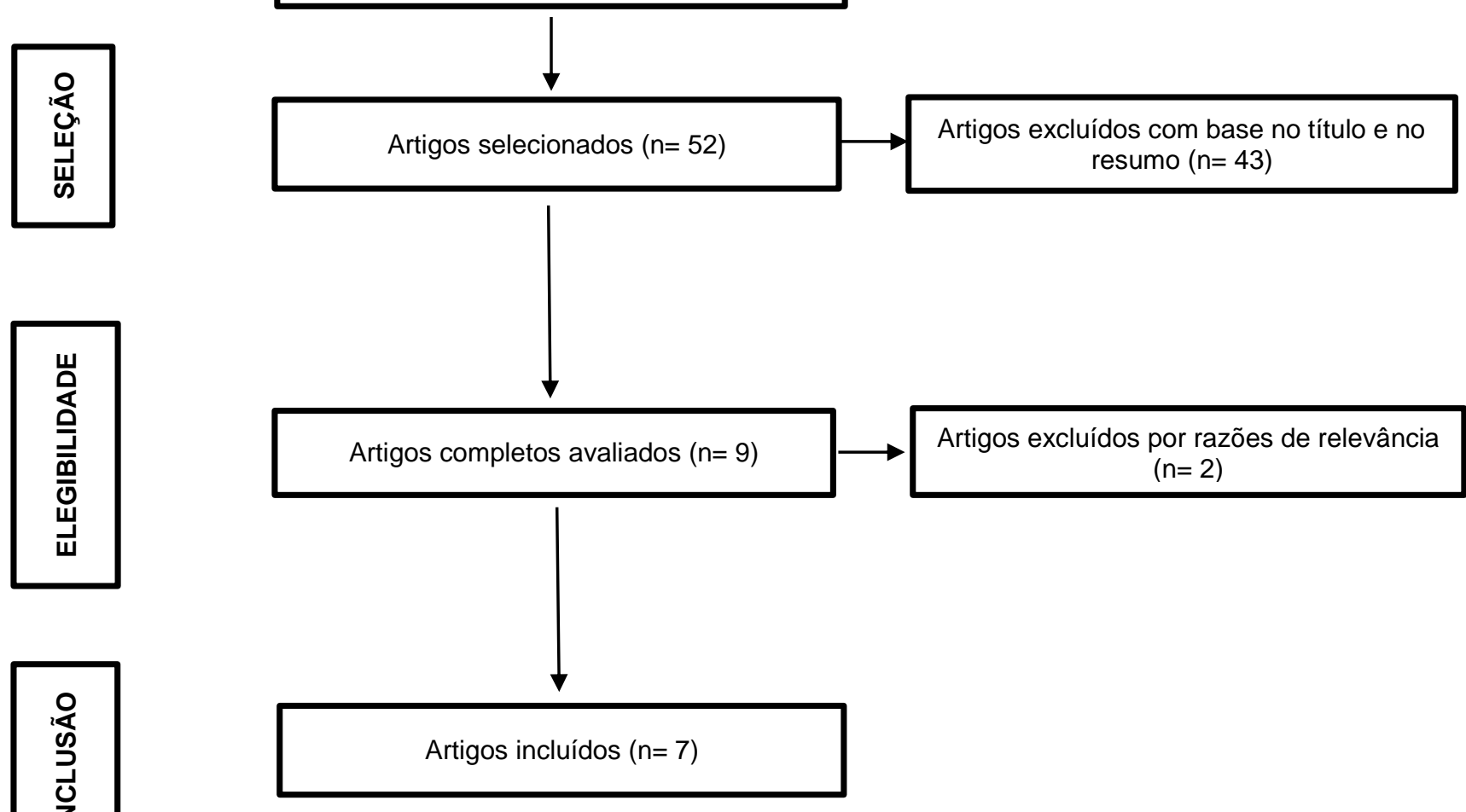

$(n=2)$

Fonte: Carneiro LV, et al., 2021.

\section{RESULTADOS}

As buscas nas bases de dados resgataram um total de 54 artigos, dos quais foram incluídos na amostra apenas 7. No que concerne ao tipo de pesquisa realizada, observou-se a predominância da abordagem qualitativa.

Em relação ao período de publicação, identificou-se que todos os artigos foram publicados entre 2012 e 2020. Quanto à origem das pesquisas, os artigos utilizados na presente revisão são de estudos desenvolvidos no Brasil $(n=5)$, Austrália $(n=1)$ e Zimbábue $(n=1)$. No tocante aos idiomas, a maioria dos artigos foram publicados em português $(n=5)$.

Dentre os artigos selecionados, constatou-se que os desafios no processo de educação inclusiva para crianças com TEA são comuns tanto no contexto nacional, como internacional, e estão relacionados principalmente a características estruturais das escolas, carência de tecnologia de ensino adequada e falta de qualificação dos professores para atenderem esse público.

Os artigos foram classificados a partir de alguns descritores, tais como: autor/ano, objetivo, método, país de publicação e principais resultados, de maneira a organizar as informações obtidas conforme os elementos disponíveis na sua identificação (Quadro 1). 
Quadro 1 - Características gerais dos artigos selecionados para a amostra.

\begin{tabular}{|c|c|c|c|c|c|}
\hline № & Autor/Ano & Objetivo & Método & País & Principais resultados \\
\hline 1 & $\begin{array}{l}\text { CAMARGO SPH, et al. } \\
\qquad(2020)\end{array}$ & $\begin{array}{l}\text { Investigar as principais dificuldades, os desafios } \\
\text { e as barreiras enfrentadas por professores de } \\
\text { alunos com diagnóstico médico prévio de TEA } \\
\text { em situação de inclusão na escola comum. }\end{array}$ & Qualitativa & Brasil & $\begin{array}{l}\text { As professoras fizeram referência às } \\
\text { dificuldades de se relacionar com os } \\
\text { alunos com TEA e da dificuldade deles em } \\
\text { se relacionar com os colegas. }\end{array}$ \\
\hline 2 & HODGES A, et al. (2019) & $\begin{array}{l}\text { Analisar as perspectivas de pais e educadores } \\
\text { sobre as experiências e desafios que impactam } \\
\text { a participação de alunos com TEA na escola } \\
\text { primária. }\end{array}$ & Qualitativa & Austrália & $\begin{array}{l}\text { Os educadores informaram que muitas } \\
\text { estratégias poderiam ser utilizadas em } \\
\text { sala de aula e que não são implementadas } \\
\text { devido à falta de tempo e recursos. }\end{array}$ \\
\hline 3 & $\begin{array}{l}\text { LIMA SM e LAPLANE } \\
\text { ALF (2016) }\end{array}$ & $\begin{array}{l}\text { Analisar o acesso e a permanência de alunos } \\
\text { com autismo na escola e verificar quais os } \\
\text { apoios terapêuticos e educacionais aos quais } \\
\text { eles tiveram acesso. }\end{array}$ & Quantitativa & Brasil & $\begin{array}{l}\text { A análise das trajetórias escolares de } \\
\text { alunos com autismo indicou que há uma } \\
\text { grande evasão escolar. }\end{array}$ \\
\hline 4 & MAJOKO T (2016) & $\begin{array}{l}\text { Investigar barreiras sociais e facilitadores de } \\
\text { inclusão de crianças com TEA no ensino } \\
\text { primário regular no Zimbábue, de acordo com as } \\
\text { perspectivas e experiências de professores. }\end{array}$ & Qualitativa & Zimbábue & $\begin{array}{l}\text { Os professores relataram que sem } \\
\text { treinamento no serviço, era um desafio } \\
\text { tentar incluir crianças com TEA na sala de } \\
\text { aula. }\end{array}$ \\
\hline 5 & $\begin{array}{l}\text { PIMENTEL AGL e } \\
\text { FERNANDES FDM } \\
\qquad(2014)\end{array}$ & $\begin{array}{l}\text { Identificar e descrever as dificuldades e o valor } \\
\text { atribuído ao trabalho com crianças com autismo, } \\
\text { por professores. }\end{array}$ & Quanti-Quali & Brasil & $\begin{array}{l}\text { Os professores consideraram que a } \\
\text { escola oferece apoio suficiente para seu } \\
\text { trabalho, mas que há pouca contribuição } \\
\text { de outros profissionais e falta de } \\
\text { tecnologia de ensino adequada. }\end{array}$ \\
\hline 6 & $\begin{array}{l}\text { RODRIGUES IB, et al. } \\
(2012)\end{array}$ & $\begin{array}{l}\text { Compreender quais as concepções dos } \\
\text { professores da rede pública sobre os alunos } \\
\text { diagnosticados como portadores de TGD ou } \\
\text { autismo no ensino regular e que posições } \\
\text { assumem perante os alunos com base em tais } \\
\text { concepções. }\end{array}$ & Qualitativa & Brasil & $\begin{array}{l}\text { As professoras informaram que por causa } \\
\text { da dificuldade de a escola lidar com os } \\
\text { alunos com TEA, ocorre a } \\
\text { responsabilização do professor pela } \\
\text { estada do "aluno-problema" no período de } \\
\text { aulas. }\end{array}$ \\
\hline 7 & $\begin{array}{l}\text { WEIZENMANNLS, et al. } \\
(2020)\end{array}$ & $\begin{array}{l}\text { Investigar a experiência de professores em } \\
\text { relação à inclusão de alunos com TEA, } \\
\text { contemplando sentimentos e práticas docentes. }\end{array}$ & Qualitativa & Brasil & $\begin{array}{l}\text { As professoras relataram falta de apoio e } \\
\text { de conhecimento acerca da prática } \\
\text { pedagógica com crianças com TEA. }\end{array}$ \\
\hline
\end{tabular}

Fonte: Carneiro LV, et al., 2021. 


\section{DISCUSSÃO}

Os artigos selecionados nesta revisão abordam dois eixos principais em relação a temática da educação inclusiva de crianças com TEA: o primeiro diz respeito a trajetória desses alunos na escola em termos quantitativos, analisando medidas de evasão escolar e progressão nos anos letivos - com apenas um artigo selecionado. Enquanto o segundo eixo, composto pelos demais artigos, apresenta uma abordagem qualitativa, analisando o discurso de educadores com experiências pessoais no ensino de alunos com TEA, sendo que um dos estudos também aborda a visão dos pais dessas crianças em relação ao processo educacional dos seus filhos.

Em relação ao primeiro eixo, Lima SM e Laplane ALF (2016) avaliaram a inserção de crianças com TEA na rede de ensino de um município no interior do estado de São Paulo, através de dados secundários do Censo da Educação Básica entre os anos de 2009 e 2012. Esse estudo demonstrou que nem todos os alunos com TEA de fato frequentam escolas regulares dentro da perspectiva da educação inclusiva, uma vez que mais da metade das matrículas dessas crianças ocorria em instituições de ensino especial. Mesmo dentre as matrículas no ensino regular, em algumas instituições as crianças não frequentavam as mesmas salas de aula que as crianças com desenvolvimento típico, compartilhando apenas os espaços comuns da escola nos horários de intervalo. Ademais, a evasão escolar também foi significativa, uma vez que menos de $10 \%$ das crianças permaneceu matriculada durante todos os anos estudados. Vale ressaltar, que essa problemática foi mais acentuada entre aqueles que se encontravam nas séries finais do ensino fundamental e inseridos em escolas municipais. Os autores ainda evidenciaram, que o processo de transição desses alunos da rede municipal de ensino para a estadual, contribui para o aumento da evasão escolar.

Embora não seja possível fazer considerações gerais, uma vez que apenas um estudo abordou diretamente dados estatísticos sobre adesão e evasão escolar de crianças com TEA, pode-se afirmar que apesar das garantias legais, a participação desses alunos dentro de um ambiente escolar inclusivo não é uma realidade em todos os municípios brasileiros (LIMA SM e LAPLANE ALF, 2016).

Em relação ao segundo eixo, apesar de métodos diferentes de coleta terem sido utilizados nas pesquisas, houve vários pontos recorrentes nos discursos dos educadores que atuam no ensino de crianças com TEA, sendo um dos principais temas o sentimento de despreparo que muitos profissionais experienciam (WEIZENMANN LS, et al., 2020; PIMENTEL AGL e FERNANDES FDM, 2014).

Estudo nacional evidenciou que todas as professoras entrevistadas apresentavam medo e insegurança ao se depararem pela primeira vez com crianças com TEA dentro das salas de aula (WEIZENMANN LS, et al., 2020). Outra pesquisa brasileira, destacou que os professores relataram sensação de despreparo técnico e necessidade de ampliar tecnologias e trocas de experiências para otimizar suas práticas em relação à educação inclusiva (PIMENTEL AGL e FERNANDES FDM, 2014). Entretanto, o desejo por mais cursos preparatórios e oportunidades de formação continuada também foi manifesto por outros educadores no contexto internacional (MAJOKO T, 2016; HODGES A, et al., 2019).

A declaração de Salamanca aponta caminhos para melhorar o recrutamento e treinamento de professores dentro do contexto da educação inclusiva, sugerindo um enfoque mais detalhado nos cursos de graduação em pedagogia, assim como programas de formação continuada para educadores já em exercício profissional, apontando ainda o papel das universidades como fundamental em termos de pesquisa, avaliação e produção de material técnico sobre esse tema (UNESCO, 1944). Somado a isso, no Brasil a Política Nacional de Proteção dos Direitos da Pessoa com Transtorno do Espectro Autista prevê, em uma de suas diretrizes, o incentivo à formação e capacitação de profissionais especializados, incluindo, portanto, professores e outros profissionais ligados a educação inclusiva (BRASIL, 2012).

Dentre as dificuldades para a inclusão mais comumente enfrentadas no dia-a-dia dentro das salas de aula, foram apontados tanto aspectos relacionados ao próprio aluno com TEA quanto dificuldades na própria estrutura da escola. O entendimento de que essas crianças têm características individuais particulares, exibindo diferentes graus de severidade do espectro e consequentemente necessidades educacionais únicas, foi bem compreendido por parte dos educadores, que apontaram a dificuldade de comunicação e socialização, além dos comportamentos repetitivos e a postura de isolamento social, como os principais obstáculos à inclusão dessas crianças (MAJOKO T, 2016; HODGES A, et al., 2019; CAMARGO SPH, et al., 2020). 
As salas de aula muito numerosas também foram apontadas como barreira importante, uma vez que os professores relataram dificuldade para dividir a atenção entre os alunos com TEA e os alunos com desenvolvimento típico (PIMENTEL AGL e FERNANDES FDM, 2014; CAMARGO SPH, et al., 2020). Podese afirmar, que turmas heterogêneas podem ser vistas sob uma ótica inicial como positivas à medida que estimulam a convivência com crianças com diferentes características, porém alguns educadores demonstraram preocupação com turmas com heterogeneidade excessiva, uma vez que quando há muitos casos de TEA ou outros transtornos de aprendizagem numa mesma classe, se torna mais difícil organizar atividades que incluam todas essas crianças de maneira igualitária (RODRIGUES IB, et al., 2012).

Em relação a práticas pedagógicas propriamente ditas, houve a percepção dos professores de que a educação inclusiva melhora a comunicação e as relações interpessoais de crianças com TEA, embora tenham afirmado que o processo de aprendizagem formal não consegue ser adequadamente assimilado pelo aluno (PIMENTEL AGL e FERNANDES FDM, 2014). Além disso, há também dificuldade em termos de avaliação, uma vez que muitos professores acabam por medir o aprendizado das crianças com TEA na mesma medida utilizada com crianças de desenvolvimento típico (RODRIGUES IB, et al., 2012; CAMARGO SPH, et al., 2020). O fato de que algumas crianças com TEA não atingem uma expectativa normatizada, pode gerar uma sensação de culpa/frustração no professor, que não vê seu papel de educador cumprido totalmente (WEIZENMANN LS, et al., 2020; CAMARGO SPH, et al., 2020).

O grau de aceitação e inclusão dos alunos com TEA por parte dos demais alunos foi um aspecto bastante heterogêneo nas diferentes experiências citadas. Alguns grupos relataram que não houve dificuldades para essa inclusão dentro da sala de aula, quando incentivado pelo professor, e que muitas vezes os outros alunos tinham um "carinho especial" em relação a criança com TEA (RODRIGUES IB, et al., 2012; CAMARGO SPH, et al, 2020). No entanto, houve relatos de dificuldade de inclusão fora do ambiente da sala de aula e ainda casos de bullying. Mediante a esse contexto, é fundamental que no ambiente escolar sejam desenvolvidas capacitações direcionadas a temática bullying, tanto para os professores, como para os demais alunos, visando quebrar esse paradigma existente (RODRIGUES IB, et al., 2012; MAJOKO T, 2016).

No que concerne as dinâmicas facilitadoras para a educação inclusiva, diversos educadores apontaram que a organização de rotinas e a apresentação de cronogramas estruturados e visuais durante as aulas, impactou positivamente o desempenho de alunos com TEA (MAJOKO T, 2016; HODGES A, et al., 2019; CAMARGO SPH, et al., 2020). Outrossim, também se destacou em dois estudos, que embora os educadores demonstrem proatividade em melhorar as práticas pedagógicas voltadas para crianças com TEA, falta um arcabouço técnico-teórico que respalde práticas específicas. Desse modo, faz-se necessário que os professores envolvidos nesse processo de educação, conheçam as necessidades dessas crianças, de modo a compreender quais métodos adequados devem ser utilizados em sala de aula e assim propiciar de fato uma verdadeira inclusão. Portanto, para que isso seja efetivado é crucial que os professores recebam apoio e qualificação profissional adequada (WEIZENMANN LS, et al., 2020; CAMARGO SPH, et al., 2020).

Contudo, um único grupo de entrevistados que incluiu pais de crianças com TEA denotou, a partir dos discursos, que almejam ver os filhos sendo tratados de forma igual aos demais alunos, no sentido de não haver discriminação de qualquer ordem, mas enfatizando ao mesmo tempo a importância de ações de apoio e suporte individualizados, com o intuito de suprir necessidades específicas de cada criança (HODGES A, et al, 2019). Vale ressaltar, que a inclusão da família no processo de inclusão escolar foi evidenciada por muitos professores como fundamental (PIMENTEL AGL e FERNANDES FDM, 2014; HODGES A, et al., 2019; CAMARGO SPH, et al., 2020)

Todavia, o papel dos outros profissionais de saúde foi valorizado como auxiliar no processo de inclusão escolar das crianças com TEA: desde a elaboração de laudos detalhados que permitem ao educador conhecer de forma mais aprofundada seu aluno, suas dificuldades e suas potencialidades, de forma a adaptar melhor suas práticas de ensino de forma mais individualizada, como também pelas ações e tratamentos próprios desses profissionais fora da escola, melhorando aspectos funcionais importantes dessas crianças (RODRIGUES IB, et al., 2012; MAJOKO T, 2016). Houve apenas uma experiência negativa em relação a 
esses outros profissionais, quando eles fizeram intervenções diretas dentro da sala de aula, sugerindo mudanças que os professores consideraram pouco factíveis do ponto de vista prático, levando-se em conta a sobrecarga de trabalho a que muitos educadores são submetidos (HODGES A, et al., 2019).

Nessa perspectiva, de acordo com Pimentel AGL e Fernandes FDM (2014), é de fundamental importância a efetiva inclusão das crianças com TEA no processo de ensino-aprendizagem, tendo em vista as necessidades apresentadas por esse público-alvo, bem como, pelos professores que precisam estar preparados para atuar frente à cada realidade. Portanto, destaca-se que a criança com TEA precisa de um ensino adequado e inclusivo em termos de didática e estruturação da aula, e os métodos estudados devem ser compatíveis com o alcance deste objetivo.

Durante a realização do estudo, destaca-se como limitação a incipiência de artigos científicos que contemplem os desafios para a inclusão da criança com TEA na escola regular. Por isso, recomenda-se a realização de novos estudos sobre a referida temática na perspectiva de suprir lacunas importantes nessa área.

\section{CONSIDERAÇÕES FINAIS}

O processo de inclusão escolar das crianças com TEA impõe a necessidade de adequações no ambiente escolar, no que diz respeito à estrutura física da escola e quantidade de alunos por turma, bem como no que tange à capacitação dos professores para lidarem com essas crianças e adotarem estratégias pedagógicas facilitadoras da aprendizagem das mesmas. Não adianta haver garantia de direitos de acesso à escola sem que existam condições de permanência. Portanto, diante da temática abordada, considera-se importante o conhecimento desta realidade para a discussão crítica da legislação e, especialmente, para a proposição de políticas públicas e de intervenções eficazes, visando à construção de conhecimentos, às trocas de experiências e às práticas educativas inclusivas.

\section{AGRADECIMENTOS E FINANCIAMENTO}

Agradecimento à Coordenação de Aperfeiçoamento de Pessoal de Nível Superior (Capes), pela concessão de bolsa de mestrado à Vitória Polliany de Oliveira Silva.

\section{REFERÊNCIAS}

1. ADURENS FDL, VIEIRA CM. Concepção de professores sobre a inclusão do aluno com autismo: uma pesquisa bibliográfica. Cadernos de Pós-Graduação em Distúrbios do Desenvolvimento, 2018; 18(2): 94-124.

2. BRASIL. Lei no 8.069, de 13 de julho de 1990. Estatuto da Criança e do Adolescente. Disponível em: http://www.planalto.gov.br/ccivil_03/leis/L8069.htm. Acesso em: 13 abr. 2021.

3. BRASIL. Lei 12.764, de 27 de dezembro de 2012. Institui a Política Nacional de Proteção dos Direitos da Pessoa com Transtorno do Espectro Autista. Disponível em: http://www.planalto.gov.br/ccivil_03/_ato20112014/2012/lei/112764.htm. Acesso em: 13 abr. 2021.

4. BRASIL. Ministério da educação, Instituto Nacional de Estudos e Pesquisas Educacionais Anísio Teixeira (INEP). Glossário da Educação Especial Censo Escolar, 2019.

5. BRASIL. Ministério da Saúde. Secretaria de Direitos Humanos. Avanço das políticas públicas para as pessoas com deficiência: uma análise a partir das Conferências Nacionais, 2012.

6. CAMARGO EP. Inclusão social, educação inclusiva e educação especial: enlaces e desenlaces. Ciência \& Educação, 2017; 23(1): 1-6.

7. CAMARGO SPH, et al. Desafios no processo de escolarização de crianças com autismo no contexto inclusivo: diretrizes para formação continuada na perspectiva dos professores. Educ. rev, 2020; 36: e214220.

8. CASTRO GG, et al. Inclusão de alunos com deficiências em escolas da rede estadual: um estudo sobre acessibilidade e adaptações estruturais. Revista Española de Salud Pública, 2018; 31(60): 93-106.

9. CHRISTENSEN DL, et al. Prevalence and characteristics of autism spectrum disorder among children aged 8 yearsautism and developmental disabilities monitoring network. morbidity and mortality weekly report: surveillance summaries, United States, 2016; 6(67): 1-23.

10. CUNHA E. Autismo e inclusão: psicopedagogia práticas educativas na escola e na família. 6 ed. Rio de Janeiro: Wak $\mathrm{Ed}, 2015 ; 140 \mathrm{p}$. 
11. HILL AP, et al. Epidemiology of autism spectrum disorders. In: VOLKMAR et al. (Org.). Handbook of Autism and Pervasive Developmental Disorders. v. 1. Diagnosis, Development and Brain Mechanisms. Wiley, 2014, p. 57-96.

12. HODGES A, et al. School participation: The shared perspectives of parents and educators of primary school students on the autism spectrum. Research in developmental disabilities, 2020; 97: 103550.

13. LIBERATI A, et al. The PRISMA statement for reporting systematic reviews and meta-analyses of studies that evaluate healthcare interventions. BMJ, 2009; 339: 1-28.

14. LIMA SM, LAPLANE ALF. Escolarização de Alunos com Autismo. Rev. bras. educ. espec, 2016; 22(2): $269-284$.

15. MAJOKO T. Inclusion of children with autism spectrum disorders: Listening and hearing to voices from the grassroots. Journal of autism and developmental disorders, 2016; 46(4): 1429-1440.

16. MENDES KDS, et al. Revisão integrativa: método de pesquisa para a incorporação de evidências na saúde e na enfermagem. Texto \& Contexto - Enfermagem, 2008; 17(4): 758-764.

17. UNESCO. Organização das Nações Unidas para a Educação, a Ciência e a Cultura. Declaração de Salamanca sobre princípios, política e prática na área das necessidades educativas especiais, 2014 . Disponível em: http://unesdoc.unesco.org/images/0013/001393/139394por.pdf. Acesso em: 13 abr. 2021.

18. PIMENTEL AGL, FERNANDES FDM. A perspectiva de professores quanto ao trabalho com crianças com autismo. Audiol. Commun. Res, 2014; 19(2): 171-178.

19. RODRIGUES IB, et al. Análise institucional do discurso de professores de alunos diagnosticados como autistas em inclusão escolar. Psicol. teor. Prat, 2012; 14(1): 70-83.

20. SILVA M, MULICK JA. Diagnosticando o transtorno autista: aspectos fundamentais e considerações práticas. Psicol. cienc. prof, 2009; 29(1): 116-131.

21. SOUZA MT, et al. Integrative review: what is it? how to do it? Einstein, 2010; 8(1): 102-106.

22. THIENGO ER, et al. Inclusão de alunos autistas como percursos da redução dos danos causados pelo desprezo social: uma revisão bibliográfica, Revista Eletrônica Acervo Saúde, 2021; 13(2): e4942.

23. WEIZENMANN LS, et al. Inclusão escolar e autismo: sentimentos e práticas docentes. Psicol. Esc. Educ, 2020; 24: e217841. 